\title{
Tecnura
}

ESTUDIO DE CASO

\section{Identificación de un método cuantitativo para la evaluación de la calidad ambiental de centros educativos cercanos a antenas de estaciones base de telefonía}

\section{Identification of a quantitative method for the evaluation of the environmental quality of educational centers near to antennas of base stations of mobile telephony}

\author{
Alexander Armesto Arenas ${ }^{1}$, Wilson Angarita Castilla ${ }^{2}$
}

Fecha de recepción: 10 de enero de 2016

Fecha de aceptación: 23 de noviembre de 2016

Cómo citar: Armesto A., A. y Angarita C., W. (2017). Identificación de un método cuantitativo para la evaluación de la calidad ambiental de centros educativos cercanos a antenas de estaciones base de telefonía. Revista Tecnura, 21(51), 132-139. doi: 10.14483/udistrital.jour.tecnura.2017.1.a010

\section{Resumen}

Contexto: Este estudio tiene como objetivo definir un método cuantitativo apropiado para la evaluación de la calidad ambiental relacionada con el funcionamiento de antenas de estaciones base de telefonía móvil, ubicadas cerca de centros educativos. El estudio está basado en el método de Norbert Dee de Battel Columbus Laboratories.

Método. Se tuvieron en cuenta las cantidades afectadas en el área de estudio: número de especies de flora y fauna, número de personas, niveles máximos de radiación, y la distancia permitida para la instalación de antenas en Colombia. El estudio se realizó en un colegio de la ciudad de Ocaña, situado cerca de una antena estación base de telefonía móvil. Para las pruebas se utilizaron encuestas, el software QGis, y un equipo de medición de radiación electromagnética de alta frecuencia (Spectran HF 60105. Aronia).

Resultados: Se obtuvieron seis ecuaciones que permitieron definir el índice de calidad ambiental de acuerdo con la relación matemática entre las cantidades afectadas y los límites de ubicación y radiación electromagnética de las antenas de telefonía móvil.

Conclusiones: El método cuantitativo propuesto permite medir índices de calidad ambiental en centros educativos afectados por la ubicación y el funcionamiento cercano de antenas de estaciones base de telefonía móvil; con esto es posible evitar la implementación de una evaluación subjetiva y poco específica.

Palabras clave: ambiente, antena, colegio, telefonía.

\begin{abstract}
Context: The objective of this research is to define an appropriate quantitative method to evaluate the environmental quality affected by the location of antennas of base stations of mobile telephony near educative centers. This study is based on the method of Norbert Dee from Battel Columbus Laboratories.

Method: For the development of the study, we took into account the most affected parts: number of species (both animals and plants), people, maximum
\end{abstract}

1 Ingeniero ambiental, candidato a magíster en Ingeniería Ambiental. Docente de la Universidad Francisco de Paula Santander, Ocaña, Colombia. Contacto: aarmestoa@ufpso.edu.co

2 Ingeniero ambiental, candidato a magíster en Desarrollo Sostenible y Medio Ambiente. Docente de la Universidad Francisco de Paula Santander, Ocaña, Colombia. Contacto: wangaritac@ufpso.edu.co 
levels of radiation, and the minimum allowed distance for the installation of antennas in Colombia. The study was conducted at a school in the city of Ocaña, located near an antenna base station for mobile telephony. For the tests we used surveys, the QGis software, and a high-frequency electromagnetic radiation measuring equipment (Spectran HF 60105. Aronia).

Results: As a result, we obtained six equations that help define the environmental quality index according to the mathematical relationship between the affected parts, the limit of location, and the radiation of mobile phone antennas permitted in any region.

Conclusions: The proposed method allows measuring indices of environmental quality in educational centers affected by the location and function of antennas base stations of mobile telephony. Additionally, a quantitative method prevents the need to implement a subjective and unspecific evaluation.

Keywords: antenna, environment, schools, telephony.

\section{INTRODUCCIÓN}

La sostenibilidad de las áreas urbanas tiene una estrecha relación con su calidad ambiental (Ministerio del Medio Ambiente, 2013). El medio ambiente es un sistema complejo compuesto de partes físicas, biológicas y sociales, y el uso de estas puede tener efectos adversos o benéficos. En este sentido, debe tenerse en cuenta que los métodos cuantitativos son definidos como técnicas de conteo, de medición y de razonamiento abstracto (Medina, 2001). Una técnica para evaluar este problema, como primer paso, es convertir estos parámetros en unidades acordes con indicadores de calidad ambiental (Dee et al., 1972). Con respecto a las alteraciones por la instalación de antenas de telefonía móvil cercanas a centros educativos, esta metodología permitirá, en forma más clara y real, medir y comparar las partes afectadas con el todo del lugar de estudio.

En ese sentido, hay que considerar que este tipo de tecnologías ha tenido mucho florecimiento desde los años 1970 (Martínez, 2001), en especial en Latinoamérica (Gasca, Camargo y Medina, 2014), en donde su funcionamiento consiste en enviar y recibir llamadas por medio de ondas electromagnéticas en una determinada área (Fernández, González y Rubio, 2002) emitidas en una establecida banda de frecuencia del espectro electromagnético (Cadena, Eslava y Franco, 2015). Este tipo de avances funciona con equipos denominados "antenas" (Anguera y Pérez, 2008). La radiación electromagnética corresponde a ondas eléctricas y magnéticas que se disipan en el espacio a la velocidad de la luz (Ornetta, s.f.) y forma parte de todo nuestro entorno, incluyendo los seres vivos (García, 2005). Su relación y sus efectos poco se han estudiado (Torres y Osorio, 2007). Existen estudios que relacionan varias enfermedades con este tipo de contaminación (Tchernitchin y Riveros, 2004). La Organización Mundial de la Salud (OMS), a través de la Agencia Internacional de Investigación del Cáncer (IARC), cataloga la radiación electromagnética emitida por antenas de telefonía móvil y Wi-Fi como cancerígenos (De la Rosa, 2014). Lamentablemente los seres humanos están sufriendo graves consecuencias y no se han unificado respuestas al respecto (Bergareche, 2013). Debido a esto y con el fin de proteger a los seres vivos, organizaciones internacionales ya están investigando (Torres y Alzate, 2006). De hecho, hay metodologías que permiten analizar los lugares más alterados por la radiación (Balmori, 2006). Algunos países para protegerse han implementado el principio de precaución y otras organizaciones han unido fuerzas conjuntas para estudiar y minimizar los riesgos de exposición a radiación no ionizante (Secretaría de Medio Ambiente y Salud Laboral de Catalunya, 2010). En nuestro país ya se definió una distancia mínima para la ubicación de antenas de 
telefonía móvil cerca de centros educativos, centros geriátricos y jardines infantiles (Ministerio de Salud y Protección Social-Organización Panamericana de Salud, 2016). Acorde a lo anterior, este artículo presenta métodos cuantitativos para el respectivo estudio de calidad ambiental con el fin de mejorar la evaluación de las alteraciones ambientales con respecto al funcionamiento de antenas de estaciones base de telefonía móvil, cuando están cerca de escuelas, colegios u universidades.

\section{METODOLOGÍA}

Para la aplicación de los respectivos métodos cuantitativos se tuvieron en cuenta los límites máximos y mínimos permitidos sobre radiación y ubicación de antenas de estaciones base de telefonía móvil, y las respectivas cantidades afectadas. En cuanto a la implementación y toma de datos se dispuso un equipo de medición electromagnética de alta frecuencia Spectran HF-60105 Aronia, software QGis 2.8.1, Google Earth, Google SketchUp, y visitas a la zona afectada. Con respecto a la medición de la radiación electromagnética, se tomó el máximo valor registrado durante seis minutos. Como lugar de prueba se escogió el Instituto Técnico Industrial Ocaña (ITI), ubicado en la coordenadas latitud $8^{\circ}$ $15^{\prime} 27.02^{\prime \prime} \mathrm{N}$, longitud $73^{\circ} 21^{\prime} 29.85^{\prime \prime} \mathrm{O}$.

\section{RESULTADOS}

Los componentes bióticos y abióticos interactúan entre sí y cualquier cambio que se produzca en el medio abiótico repercutirá sobre los seres vivos. A partir de esta afirmación se puntualizó sobre cuatro componentes ambientales (tabla 1) de los centros educativos.

Los índices de calidad ambiental (ICA) de cada uno de los indicadores se valoraron de 0 a 1 (tabla 2).

A los índices de calidad ambiental de cada una de las variables de los componentes ambientales se les determinó una ecuación (tabla 3) que relacionara las partes afectadas con la calidad ambiental.

Tabla 1. Componentes ambientales

\begin{tabular}{ll}
\hline \multicolumn{1}{c}{ Componente ambiental } & \multicolumn{1}{c}{ Indicadores } \\
\hline Ecología & Área de flora y fauna afectada por la radiación electromagnética de telefonía móvil. \\
\hline Contaminación & Contaminación atmosférica por radiación electromagnética. \\
\hline Componentes bióticos del medio & $\begin{array}{l}\text { Flora: número de especies afectadas. } \\
\text { Fauna: número de especies afectadas. }\end{array}$ \\
\hline \multirow{2}{*}{ Social } & $\begin{array}{l}\text { Población urbana relacionada con gestión ambiental. } \\
\text { Área afectada "usos de suelo". } \\
\text { Ubicación de la antena de acuerdo con la población afectada. }\end{array}$ \\
\hline
\end{tabular}

Fuente: elaboración propia.

Tabla 2. Valoración de la calidad ambiental

\begin{tabular}{cl}
\hline ICA & Calidad ambiental \\
\hline$<\mathbf{0 , 2}$ & Muy baja calidad ambiental \\
\hline $\mathbf{( 0 , 2}-\mathbf{0 , 4}]$ & Baja calidad ambiental \\
\hline $\mathbf{( 0 , 4 - \mathbf { 0 } , 6 ]}$ & Calidad ambiental media \\
\hline $\mathbf{( 0 , 6 - 0 , 8 ]}$ & Calidad ambiental alta \\
\hline $\mathbf{( 0 , 8 - 1 ]}$ & Calidad ambiental muy alta \\
\hline
\end{tabular}

Fuente: Ministerio de Medio Ambiente (2013). 
Tabla 3. Ecuaciones por indicador ambiental

\begin{tabular}{|c|c|c|}
\hline Indicador ambiental & $\begin{array}{c}\text { ICA } \\
\text { Ecuación }\end{array}$ & Descripción \\
\hline $\begin{array}{l}\text { Área de flora, fauna } \\
\text { y población humana } \\
\text { afectada }\end{array}$ & $\begin{array}{c}A=\pi r^{2} \\
I C A=\frac{\text { Area afectada }}{\text { Area de estudio }}+1\end{array}$ & $\begin{array}{l}\text { Cuanta más área de cobertura tenga el punto } \\
\text { crítico de la fuente, menor será el ICA. }\end{array}$ \\
\hline $\begin{array}{l}\text { Contaminación atmos- } \\
\text { férica por radiación } \\
\text { electromagnética }\end{array}$ & $I C A=\frac{\cos \left(\frac{\pi \mathrm{I}}{\operatorname{Im} a x}\right)+1}{2}$ & $\begin{array}{l}\text { Las mediciones de intensidad (I) muestran } \\
\text { valores muy pequeños (entre nanowatts } / \mathrm{m}^{2} \\
\text { y miliwatts } / \mathrm{m}^{2} \text { ) y la disminución del ICA es } \\
\text { muy lenta con relación a la intensidad de la } \\
\text { radiación, mostrando una curva sinusoidal. }\end{array}$ \\
\hline $\begin{array}{l}\text { Número de especies } \\
\text { afectadas de fauna y } \\
\text { flora }\end{array}$ & $I C A=-\frac{\mathrm{N}^{\circ} \text { de especies afectadas }}{\mathrm{N}^{\circ} \text { de especies en total }}+1$ & $\begin{array}{l}\text { En cuanto a los componentes bióticos del } \\
\text { medio, cuanto mayor es el número de espe- } \\
\text { cies afectadas por la radiación, menor es el } \\
\text { ICA de la zona de estudio. }\end{array}$ \\
\hline $\begin{array}{l}\text { Número de personas } \\
\text { afectadas }\end{array}$ & $I C A=-\frac{\mathrm{N}^{\circ} \text { de personas afectadas }}{\mathrm{N}^{\circ} \text { total de personas }}+1$ & $\begin{array}{l}\text { Cuanto mayor sea el número de personas } \\
\text { afectadas por las antenas, menor será el ICA. }\end{array}$ \\
\hline $\begin{array}{l}\text { Ubicación de la antena } \\
\text { de acuerdo con la } \\
\text { población afectada }\end{array}$ & $\begin{array}{c}I C A=\left\{\begin{array}{l}\frac{0.4 x}{x_{\min }}, x<x_{\min } \\
\frac{0.6\left(x-x_{\min }\right)}{x_{\min }}+0.4, \mathrm{x}_{\min }<x<x_{\max } \\
\text { Xmáx: distancia máxima } \\
\text { Xmín: distancia mínima }\end{array}\right. \\
\quad \mathrm{x}: \text { distancia antena-centro educativo }\end{array}$ & $\begin{array}{l}\text { A menor distancia de la antena, habrá me- } \\
\text { nor ICA. }\end{array}$ \\
\hline
\end{tabular}

Fuente: elaboración propia.

La relación entre la sumatoria total de cada uno de los ICA y la sumatoria de la calificación máxima que estos pueden tener es el valor que determina la puntuación general de la calidad ambiental del sitio de estudio (ecuación (1)).

$$
I C A=\frac{\sum I C A_{i}}{\sum I C A_{\max }}
$$

El primer paso es hacer un diagnóstico que permita conocer las cantidades de las partes alteradas del sitio de estudio (figura 1).
La descripción del colegio (tabla 4) muestra una extensa superficie arbórea, de planta física y sitios de recreación.

Para la medición de la radiación electromagnética (figura 2) se tomaron tres puntos: sitio de recreación (canchas), salón de clases y otro en la cafetería. 


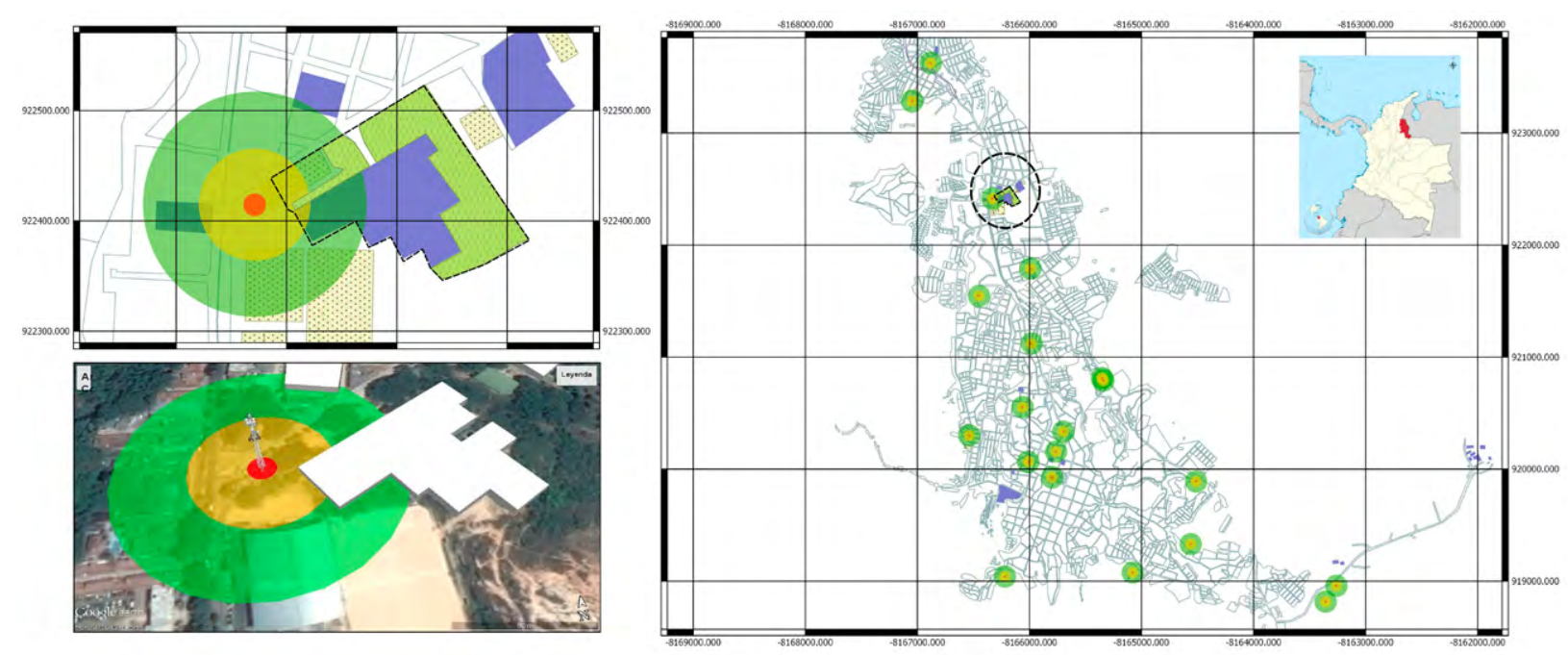

Figura 1. Ubicación Instituto Técnico Industrial

Fuente: elaboración propia.

Tabla 4. Descripción cuantitativa del colegio ITI

\begin{tabular}{|c|c|c|c|c|c|c|c|}
\hline \multirow{2}{*}{$\begin{array}{l}\text { Área total } \\
\quad\left(\mathbf{m}^{2}\right)\end{array}$} & \multirow{2}{*}{$\begin{array}{l}\text { Área total de } \\
\text { flora y fauna } \\
\text { urbana }\left(\mathrm{m}^{2}\right)\end{array}$} & \multirow{2}{*}{$\begin{array}{c}\text { Área física } \\
\left(\mathbf{m}^{2}\right)\end{array}$} & \multirow{2}{*}{$\begin{array}{l}\text { Número de } \\
\text { especies de } \\
\text { flora urbana }\end{array}$} & \multirow{2}{*}{$\begin{array}{l}\text { Número de } \\
\text { especies de } \\
\text { fauna urbana }\end{array}$} & \multirow{2}{*}{$\begin{array}{l}\text { Población total } \\
\text { (estudiantes y } \\
\text { administrativos) }\end{array}$} & \multicolumn{2}{|c|}{ Antenas de telefonía móvil instaladas } \\
\hline & & & & & & Cantidad Cerca & Dentro Distancia (m) \\
\hline 22618.19 & 8802.15 & 8876.917 & 11 & 11 & 950 & $\mathrm{X}$ & 40 \\
\hline
\end{tabular}

Fuente: elaboración propia.

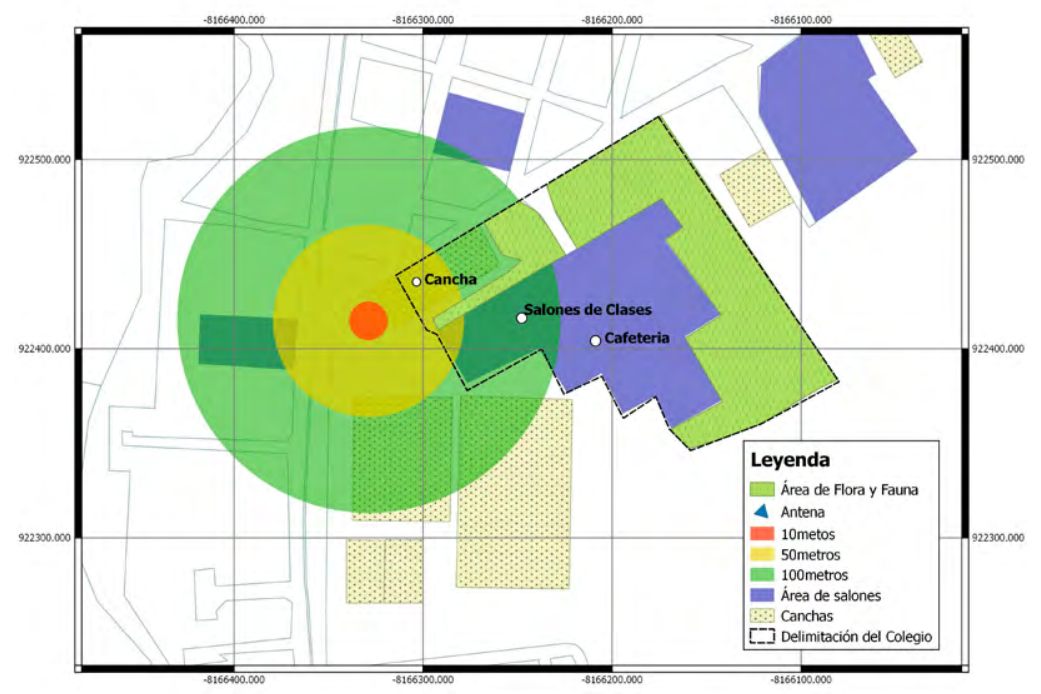

Figura 2. Puntos de medición de radiación electromagnética

Fuente: elaboración propia. 
Todas las mediciones mostraron valores inferiores al máximo establecido por Colombia, describiendo mayor valor la medición realizada en las canchas (tabla 5).

Una vez determinado lo anterior, se establece la calidad ambiental general. De acuerdo con el sitio de estudio escogido, el índice de calidad ambiental del lugar de estudio fue 0,45 (tabla 6), representado una calidad ambiental media.

Tabla 5. Intensidad de radiación en cada punto de medición

\begin{tabular}{llcc}
\hline $\begin{array}{l}\text { Banda de frecuencia } \\
(\mathbf{M H z})\end{array}$ & Lugar de medición & Intensidad $\mathbf{( W / \mathbf { m } ^ { 2 } )}$ & $\begin{array}{c}\text { Intensidad máxima } \\
\text { permitida W/m }\end{array}$ \\
\hline \multirow{2}{*}{1900} & Canchas & $2.45 \times 10^{-7}$ & \multirow{2}{*}{9.5} \\
\cline { 2 - 3 } & Salón de clases & $7.76 \times 10^{-8}$ & \multirow{2}{*}{} \\
\cline { 2 - 3 } & Cafetería & $6.94 \times 10^{-9}$ & \\
\hline
\end{tabular}

Fuente: elaboración propia.

Tabla 6. Índice de calidad ambiental del colegio ITI con respecto a la ubicación y funcionamiento de antenas de telefonía móvil

\begin{tabular}{|c|c|c|c|c|c|c|}
\hline $\begin{array}{c}\text { Componente } \\
\text { ambiental }\end{array}$ & Variables & $\mathbf{Q}$ & Q Mín. & Q Máx. & ICA & $\begin{array}{c}\text { Calidad } \\
\text { ambiental }\end{array}$ \\
\hline Ecología & $\begin{array}{l}\text { Área de flora y fauna afec- } \\
\text { tada }\left(\mathrm{m}^{2}\right)\end{array}$ & 833.76 & - & 8802.15 & 0.90 & Muy alta \\
\hline Contaminación & $\begin{array}{l}\text { Intensidad de radiación } \\
\text { electromagnética }\left(\mathrm{w} / \mathrm{m}^{2}\right)\end{array}$ & $2.4 \times 10^{-7}$ & - & 9.5 & 1 & Muy alta \\
\hline \multirow{2}{*}{$\begin{array}{l}\text { Componentes bióti- } \\
\text { cos del medio }\end{array}$} & $\begin{array}{l}\text { Flora: número de especies } \\
\text { afectadas }\end{array}$ & 4 & - & 11 & 0.6 & Media \\
\hline & $\begin{array}{l}\text { Fauna: número de especies } \\
\text { afectadas }\end{array}$ & 11 & - & 11 & 0 & Muy baja \\
\hline \multirow{3}{*}{ social } & $\begin{array}{l}\text { Número de habitantes } \\
\text { afectados }\end{array}$ & 950 & - & 950 & 0 & Muy baja \\
\hline & Área afectada & 6628.35 & - & 22618.19 & 0.7 & Alta calidad \\
\hline & $\begin{array}{l}\text { Ubicación de la antena } \\
\text { (distancia en metros) }\end{array}$ & 0 & 100 & 1000 & 0 & Muy baja \\
\hline & 3.2 & \multirow{2}{*}{ Media } \\
\hline \multicolumn{5}{|c|}{ Índice de calidad ambiental } & 0.45 & \\
\hline \multicolumn{7}{|c|}{$\begin{array}{l}\text { Q: Cantidad medida en la zona de estudio. } \\
\text { Q mín.: Valor mínimo o permitido de cada una de las variables analizadas. } \\
\text { Q máx.: Define el valor máximo o total de la variable analizada. } \\
\text { El número de especies afectadas hace referencia a las especies que permanecen en la zona crítica }(r<100 \mathrm{~m}) \text {. }\end{array}$} \\
\hline
\end{tabular}

Fuente: elaboración propia. 


\section{CONCLUSIONES}

El método cuantitativo posibilita medir el índice de calidad ambiental de acuerdo con la realidad de las cantidades de las partes de los centros de educativos afectados por la ubicación y funcionamiento cercano de antenas de estaciones base telefonía móvil, y evita del mismo modo implementar una evaluación subjetiva y poco específica.

\section{REFERENCIAS BIBLIOGRÁFICAS}

Anguera, J. y Pérez, A. (2008). Teoría de antenas. Guía de estudio. Barcelona: Universidad Ramon Llull. Recuperado de: http://web.salleurl.edu/ jaumean/ Teoria-Antenas-by-Jaume Anguera.pdf

Balmori, A. (2006). Efectos de las radiaciones electromagnéticas de la telefonía móvil sobre los insectos. Ecosistemas, 15(1), 87-95.

Bengt, K. (1996). Radiaciones no ionizantes. En: Enciclopedia de Salud y Seguridad en el Trabajo (cap. 49, pp. 1-36). Recuperado de: http://www.insht.es/ InshtWeb/Contenidos/Documentacion/TextosOnline/EnciclopediaOIT/tomo2/49.pdf

Bergareche, R.L. (2013). Contaminación por radiación electromagnética en personas vulnerables: tutela preventiva y generación de otras fuentes de energía. Actualidad Jurídica Ambiental. Recuperado de: http://www.actualidadjuridicaambiental.com/ wp-content/uploads/2013/11/2013_10_28_Raquel_Luquin_Contaminacion-electromagnetica.pdf

Cadena M., E.; Eslava B., H J. y Franco C., J.A. (2015). Gestión del espectro radioeléctrico en Colombia. Revista Tecnura, 19(45), 159. https://doi. org/10.14483/udistrital.jour.tecnura.2015.3.a12

De la Rosa, R. (2014). La enfermedad silenciada. Madrid, España: Ediciones I. Recuperado de: http:// www.escuelasinwifi.org/

Dee, N.; Baker, J.; Drobny, N.; Duke, K. y Fahringer, D.C. (1972). Description of the environmental evaluation system (EES). En: Environmental Evaluation Water Resource Planning (pp. 6-10). Recuperado de: https://babel.hathitrust.org/cgi/pt?id=coo.3192 4004263087; view=1 up;seq=22
Fernández L., A.; González L. y Rubio L., A. (2002). Telefonía móvil. Transmisión y redes de datos. Recuperado de: http://www.uhu.es/fernando.gomez/ transydat_archivos/Movil.PDF

García, S.I. (2005). La salud humana y los campos electromagnéticos de frecuencia extremadamente baja (CEM-FEB). Buenos Aires: Asociación de Toxicología Argentina. Recuperado de: http://www.ataonline.org.ar/bibliotecavirtual/documentos_utilies/ cem_feb.pdf

Gasca M., M.C.; Camargo A., L.L. y Medina D., B. (2014). Metodología para el desarrollo de aplicaciones móviles. Revista Tecnura, 18(40), 20-35. Recuperado de: http://tecnura.udistrital.edu.co/ojs/ index.php/revista/article/view/767

Martínez, E. (2001). La evolución de la telefonía móvil. Revista Red, 1, 1-6. Recuperado de: http://www. eveliux.com/mx/La-evolucion-de-la-telefonia-movil.html

Medina B., C.I. (2001). Paradigmas de la investigación sobre lo cuantitativo y lo cualitativo. Ciencia e Ingenieria Neogranadina,10, 1-13. http://www.redalyc.org/articulo.oa?id=91101010

Ministerio de Medio Ambiente (2013). Índice de calidad ambiental urbana (ICAU). Bogotá, Colombia.

Ministerio de Salud y Protección Social-Organización Panamericana de Salud (2016). Recomendaciones con respecto a la exposición por campos electromagnéticos generados por la red de telefonía móvil. En: Guía para la gestión de las DTS en el desarrollo de los procesos de promoción, prevención, vigilancia y control sanitario relacionados con los campos electromagnéticos generados por la telefonía móvil (pp. 80-83). Bogotá, Colombia. Recuperado de: https://www.minsalud.gov.co/sites/rid/Lists/BibliotecaDigital/RIDE/VS/PP/SA/gestion-dts-camposelectromagneticos-telefonia-movil.pdf

Ornetta, V.C. (s.f.). La telefonía móvil y su salud. Recuperado de: http://www.who.int/peh-emf/publications/en/esp_mobphonehealthbk.pdf

Secretaría de Medio Ambiente y Salud Laboral de Catalunya (2008). El futuro de las radiaciones no ionizantes. In amb tu + prevenció. Cuanderno preventivo: Radiaciones no ionizantes (p. 52). 
Catalunya. Recuperado de: http://www.ladep.es/ ficheros/documentos/Cuaderno Preventivo Radiaciones no ionizantes. UGT de Catalunya. 2008. pdf

Tchernitchin, N.A. y Riveros, R. (2004). Efectos de la radiación electromagnética sobre la salud. Cuadernos Medico Sociales, 44(4), 221-234. Recuperado de: http://cms.colegiomedico.cl/Magazine\%5C2004\%5C44\%5C4\%5C44_4_5.pdf
Torres, J.I. y Alzate, L.H. (2006). Efectos de las radiaciones electromagnéticas no ionizantes en sistemas biológicos. Revista Médica de Risaralda, 12(2), 44-54.

Torres, J.I. y Osorio, M.C.O. (2007). Criterios técnico-ambientales para el análisis del riesgo por contaminación electromagnética no ionizante en Colombia. Revista Luna Azul, 24, 8-14. Recuperado de: http:// www.redalyc.org/pdf/3217/321727226002.pdf 